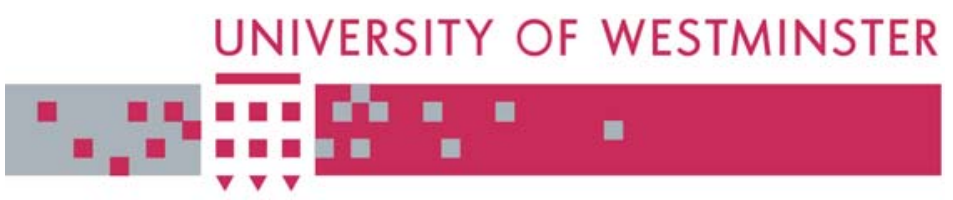

\title{
WestminsterResearch
}

http://www.wmin.ac.uk/westminsterresearch

\section{Agent-based models for community care systems analysis and design.}

Wei Huang ${ }^{1}$

Elia El-Darzi ${ }^{1}$

Panagiotis Chountas ${ }^{1}$

Peng Liu ${ }^{2}$

${ }^{1}$ Harrow School of Computer Science, University of Westminster

2 School of Information Management and Engineering, Shanghai University of Finance and Economics

Copyright (C) [2006] IEEE. Reprinted from the Proceedings of the 3rd International IEEE Conference on Intelligent Systems, pp. 278-283.

This material is posted here with permission of the IEEE. Such permission of the IEEE does not in any way imply IEEE endorsement of any of the University of Westminster's products or services. Internal or personal use of this material is permitted. However, permission to reprint/republish this material for advertising or promotional purposes or for creating new collective works for resale or redistribution must be obtained from the IEEE by writing to pubs-permissions@ieee.org. By choosing to view this document, you agree to all provisions of the copyright laws protecting it.

The WestminsterResearch online digital archive at the University of Westminster aims to make the research output of the University available to a wider audience. Copyright and Moral Rights remain with the authors and/or copyright owners.

Users are permitted to download and/or print one copy for non-commercial private study or research. Further distribution and any use of material from within this archive for profit-making enterprises or for commercial gain is strictly forbidden.

Whilst further distribution of specific materials from within this archive is forbidden, you may freely distribute the URL of the University of Westminster Eprints (http://www.wmin.ac.uk/westminsterresearch).

In case of abuse or copyright appearing without permission e-mail wattsn@wmin.ac.uk. 


\title{
Agent-based Models for Community Care Systems Analysis and Design
}

\author{
Wei Huang ${ }^{1}$, Elia El-Darzi ${ }^{1}$, Panagiotis Chountas ${ }^{1}$ and Peng Liu ${ }^{2}$
}

\begin{abstract}
In recent years, the providers of public and private sector health care services have been faced with some radical changes in the society they serve, and more importantly, development in the way that traditional health care is delivered to Information Technology (I.T) based communities. It is widely believed by health care professionals that the better health care results really come from the improved healthcare systems and more effective health care services' management. This paper focuses on using an agentbased software engineering approach and design models to the development of an appropriate agent-based healthcare software system is described in which software researchers collaborate with environment builders to enhance the levels of cooperation and support provided within an integrated agentbased community healthcare system.
\end{abstract}

Keywords - agent technology, agent-oriented software engineering, agent-based software design models, agentbased community care.

\section{INTRODUCTION}

Why agent technology? Why this new approach is suitable for the community health care domain and why it can be used to contribute to the community care service field through reorganization of the health and social care services?

Agents are artificial intelligent entities or sub systems within a dynamic environment, which can sense and act (not necessarily in that order). In 1995, Wooldridge and Jennings[15,16]have described that "An agent is a computer system situated in some environment, and that is capable of autonomous action in this environment in order to meet its design objectives."[16]. This means that agents are not isolated entities or systems, and that they are able to communicate and collaborate with other entities that are based on Distributed Artificial Intelligence (DAI) and sharing Distributed Computing (DC) communities.

This work is supported by Heath Care Computing Group, University of Westminster, London, United Kingdom.

${ }^{1}$ Harrow School of Computer Science, University of Westminster Northwick Park, Watford Road, HA1 3TP Greater London, United Kingdom Email:\{huangw, eldarze,chounp\}@wmin.ac.uk

2 School of Information Management and Engineering, Shanghai University of Finance and Economics, Shanghai, 200433, P.R. China, liupeng@mail.shufe.edu.cn
Community health care is dynamic, complex and progressive. $[1,2,3]$ Its aim is to provide such services as are necessary to maintain people and quality of life in the community when they are unable to provide for themselves, thus maintaining their independence. It is also provided by a wide range of disparate, independent organizations and agencies, typically each having their own objectives, of which the provision of community care is only a part. The objective is perfectly matched with agents' skills, capabilities, and the design goals to integrate services with their responsibilities in a coherent and efficient manner [4]

The use of agent technology for health care software system design and for community care service management is prompted by several evolutionary influences which can be summarized as follows [3]:

- The desire to provide humane and effective care systems that deliver improved services to people, enhancing social interaction and enabling more effective delivery of health care.

- The ability to define high-abstraction level care management strategies by effectively linking the health care professionals into a single framework of accountability. There are many different people or organizations (medical teams, social services, etc.) that are normally involved with different priorities and various skills and responsibilities. Agent-based organisational methods allow people to operate with full managerial responsibilities[11], e.g. established practitioners, e.g. community health doctors, senior nurses, medical services planners and health advisors etc.

- The development of an in depth understanding of health information (both the data and its sources), to provide better community medicine, which includes a wide range of the related social services that are often currently neglected. It is necessary to have a lucid method and platform to allow them to communicate, negotiate, collaborate and cooperate to achieve their common domain goals. The agent-based solutions are ideal for dealing with complex issues and challenges facing in a health care community

- Agent-base care expert systems can assist medical professionals in the tasks of monitoring, detection and diagnosis from different geographic locations and regions.

- Cooperative agents can work within community structures to change the human service programme or 
social service policies, by involvement in planning, scheduling and organizing (both for formal care and informal care services), in a more effective and timely manner.

- The concept of a shared multi-agent environment permits shared supervision and greater teamwork by integrating experts from a number of groups into common service environments. This will allow patients to visit experts, doctors or care organizations by connecting to medical agents though an agent-based community care network and platform.

\section{AGENT-BASED DESIGN MODELS FOR COMMUNITY CARE SERVICES SYSTEM'S DEVELOPMENT}

In [6,7], Jennings says: "agent-oriented approaches are well suited to developing complex software systems in general and control system in particular". As yet, there are not many agent-oriented software and applications in the market. In part, this is due to the complexity of agent system development, the absence of mature techniques and the shortage of useful software tools. The commonly considered solutions are the development of the component-based software techniques for the integration of components like database software applications into agent systems and the development of the useful structures for collections of agents into teams and groups in agent architectures. The key technique for this development is Modelling. Modelling is the design of software system before coding and final implementation. Modelling is an essential part of an agent-centred system. Agent-based models play the analogous roles in software development that blueprints and other plans (site maps, elevations, models) play in the building of a skyscraper. Using a model, those responsible for a software development project's success can assure themselves that business functionality is complete and correct, that end-user needs are met, and that the program design supports requirements for scalability, robustness, security, extendibility, and other characteristics, before implementation in code renders changes difficult and expensive to make.

Agent-based community health care is a new service style which automates the process of linking constituents with their core competencies quickly and effectively on the Internet. Important new modelling aspects for developing of those kinds of systems should considerate more than tradition systems [12]. Here in our research, it has been considered with following three design models: agent model, agent services model and acquaintance model.

- The agent model is mainly used to identify agent types and their "natural" attributes. It mainly creates agent types by aggregating roles, each emerging agent type can be represented as a role that combines all the aggregated roles attributes (responsibilities, activities, functions etc.).

- The service model describes agent responsibilities and services associated with each agent/role[5]. In each service model, there are four states of service: input, output, pre-conditions and post-conditions, and these must be identified before allowing them to represent constraints on services. A service model can sometimes be divided into several phases and can be decomposed into a set of methods.

- The acquaintance model is the guideline for agents' communication and cooperation. It is a graph with nodes in the graph corresponding to agent types and arc in the graph corresponding to communication pathway For example, the pathway for the NHS patients to access healthcare system can be simply illustrated as follows:

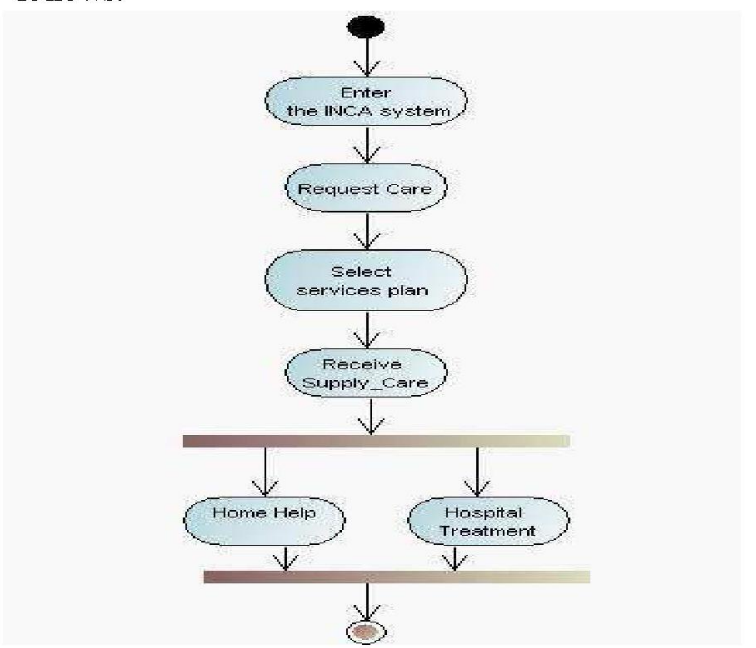

Figure 1. Pathway of Accessing Health Care System

\section{AgENT-BASEd PRACTICAL DeSIGN AND MODELLING TOOLS}

Agent-based community care unveils a tremendous range of challenges and opportunities to create more advanced distributed e-service systems by fully using agent capabilities and responsibilities, allowing us to open the door to new service styles which lead to reduced costs, improved communications and affect the way we live, work and do business.

Obviously, managing complexity issues and creating reliable agent-based software is the very difficult task facing developers of large-scale embedded software systems. In order to cope with the challenges of complexity and to ensure that the construction of the model goes through the construction of a number of views of the each one only dealing with a specific aspect. It is necessary to have additional graphical views to represent the models and their associated components or elements.

Our experience to agent-oriented software engineering research suggests that incompleteness of design models and the lack of agent-based software design and development tools that allow system complexity to be electively managed is a greater obstacle. With the emergence of agent-based software paradigm, the need for tools and 
techniques to perform analysis and design of systems became apparent. Therefore, the researchers in Health Care Computing Group at University of Westminster are also proposing and exploiting new agent-based software design tools. Our design philosophy to agent-based systems is based on progressive development and decomposition of system's construction or behaviours, i.e. extending current existing methodologies, models and adapting some modelling techniques and ideas from object-oriented software engineering (since object-oriented modelling techniques are not directly applicable to agent systems, and agents are more complex than objects) $[8,10]$, providing compositional modelling tools suitable for the verification of agent-based system structure and function. Here, in order to simplify and address design and development stage clearly, a set of agent-based graphic modelling diagrams for addressing domain-level abstractions and agents behaviours are introduced and experimented into our health care project as follows:

Agent and Role Schema Diagram: This defines the agent types by aggregating roles and it also defines role of each agent and its related attributes. It is a blueprint-style description on agent and their role schema. In analysis level, this schema helps to discover individual agents and agent's attributes. This mainly describes individual agent's functions and states and abilities. Such as in [Figure 2] an agent called Care Provider is able to communicate and negotiate with other agents to provide wide range of care services.

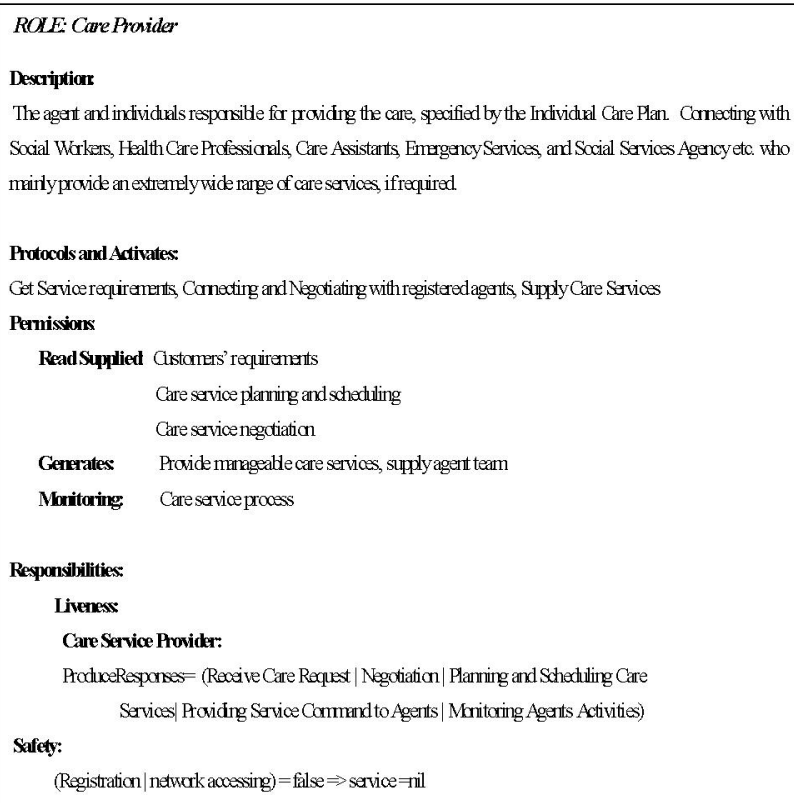

Figure 2 The agent and role model: a formal schema for agent Care Provide

Service Diagram: This provides a domain view of the services and tasks of individual agents. Those services and tasks all based on the agent's role which has described in Agent/Role Schema diagram, service diagrams also should include some additional information on practical events and particular conditions. The service may vary due to the different tasks and conditions applied to agent-based system. For example, agent Care Coordinator in event of Preparing and Updating the Individual in the event of the basic proposed services diagram for agent Care Coordinator, can be seen in [Figure 3]

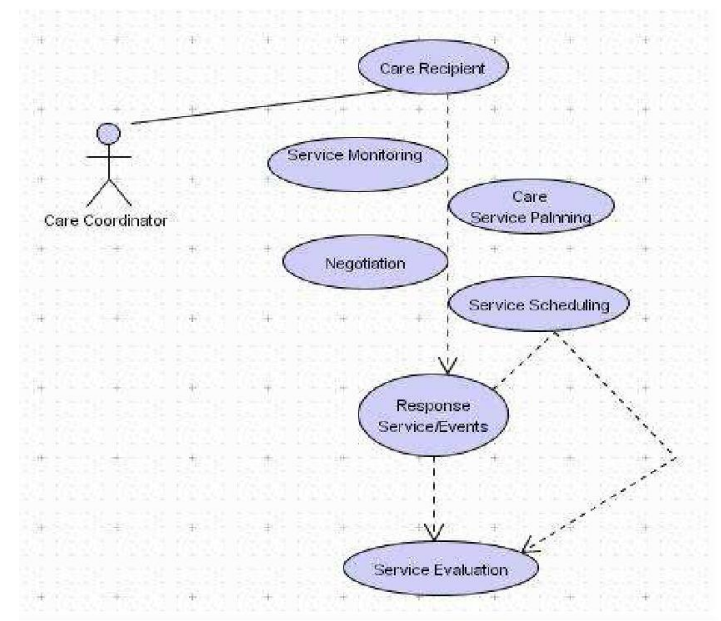

Figure 3: Service Diagram: Basic Services of Agent Care Coordinator

Collaboration and Interaction Diagram: This describes how an agent responds to the system or other agents, the communication/negotiation routing and service scheduling and planning pathway. This is normally achieved by using common communication language and protocols which are accepted by all the agents and networking systems.

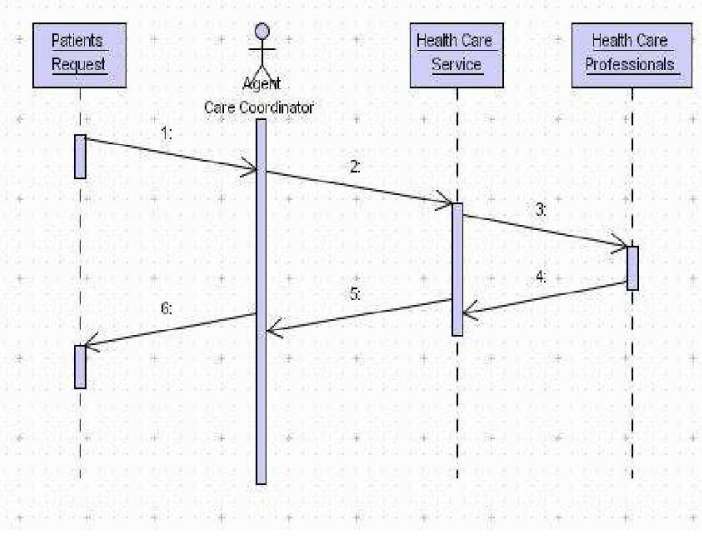

Figure4 Interaction diagram: Agent Care Coordinator interacting with health care system and patients

The diagram [Figure 4] shows the communication paths and route between the patients and health care system. It illustrates that after having carefully analysed patients' requests, the agent Care Co-ordinator passing patient's case to an appropriated health care service system, e.g. a local clinic or a dental office, hospital then those information is diverted to a responsible health care professional such as a GP or a nurse. 
Acquaintance Diagram: This defines desired agent domain behaviours of all the agents. It contains a set of use cases and the graphs corresponding communication pathway among agent. For example, as showing in [Figure 5], Agent Care Coordinator is arranging an event for a patient, it could be make an appointment with doctor or nurse or receive a prescription/ re-prescription After doctor or nurses consolidation and all the health care professionals are all connected and carefully operated following the Acquaintance diagram[Figure5].

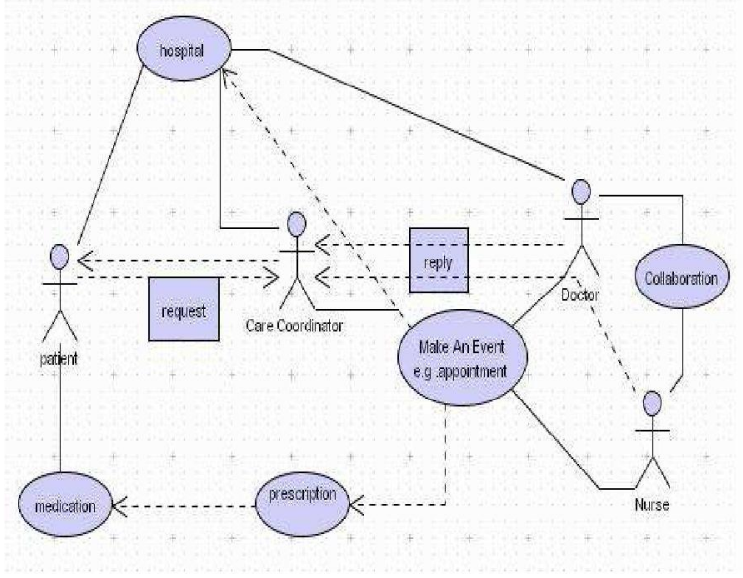

Figure5:Acquaintance diagram on event of appointments and prescription requests.

Organization Diagram: This presents the overall of the structure of an agent-oriented society, i.e. Agent-based system architecture and working environment which includes how the all the agents and it roles can be well organized and managed into a single working environment for events, whilst access database and share the resource in a planning route and interaction between the autonomous independent agencies involved in the provision of services[5].

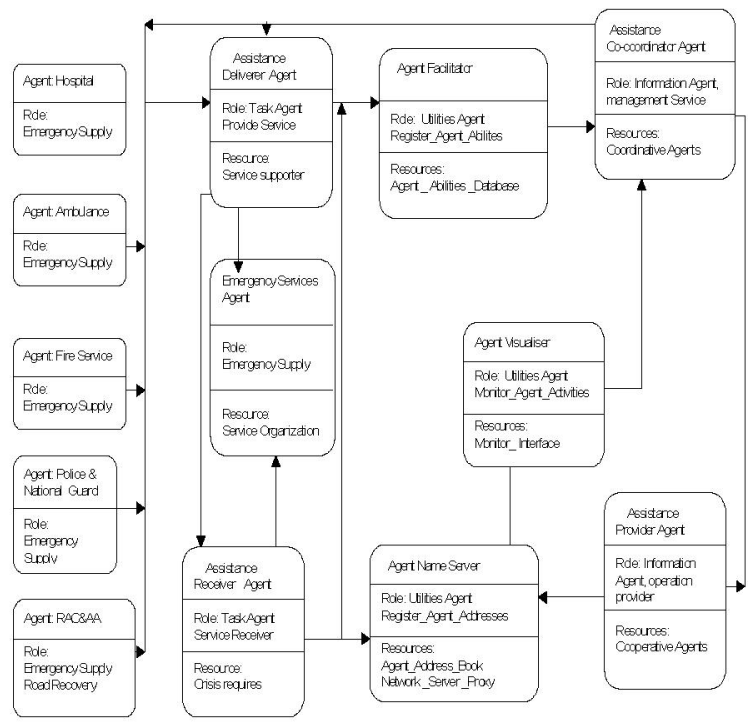

Figure 6: Organizing interactive and cooperative agents /roles in an emergency event
Organization diagram for an Agent-based Community Care System can be illustrated as follows [Figure 7]. The system can be divided into three sections, which are based on the role and functions of responsive agents: The Information Agents, i.e. Care Coordinator' Management with associated Social Care Service, are working together in the control of the system, commanding and scheduling the tasks and managing services. The User Task Interface Agents, such as GP, patients and carers, are the task providers or health care service receivers; this has a graphic interactive interface to communicate with Information Agents (Health Care Coordinator and Social Care Services). Some Task and Service Agents such as Hospital connect and cooperate with Doctors and Nurses and Care Coordinator Management Agents, and are working together. In some emergency circumstances such as transfer of patients or delivery of blood or organs, the Emergency Agents operate under the direction of the Care Coordinator Management Agents involved in providing the heath care service.

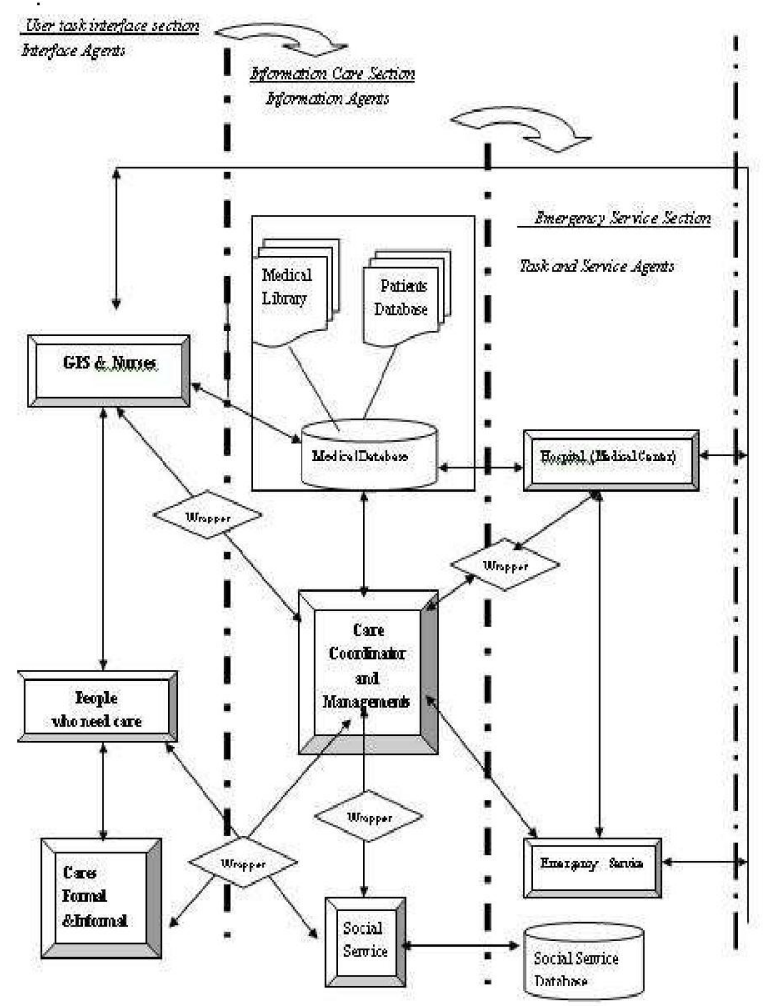

Figure 7: Organizational Diagram for a Health Care System (simplified version)

\section{THE BENEFITS OF USING INTELLIGENT AGENTS FOR COMMUNITY HEALTH CARE SYSTEMS}

Agent technology is a new research direction, which provides a range of possibilities and opportunities for software designers and developers to design and develop new agent-oriented computer software and applications.

The major advantage of agent-oriented software (and thus of intelligent agent technology) is that it allows these 
intelligent agents i.e. artificial intelligent entities, to serve as proxies for high-level abstractions of the active entities that are present in a service-based software system. Although agent-based system modelling is still in its early stages of development. But we have already discovered and experienced some positive design strengths for system design and development, such as the aspect of agent software development approach leads us to focus on using agent technology to redesign and reorganize the existing community care services and management system. Communicated and cooperative agents could carry on a wide range of activities (such as information accessing, interaction and communication, health care service planning, task scheduling, decisions making and health care resource management, etc.).

By using agent-based community care service system could provide an effective control and new management style. The benefits of using agents and agent technology for health care section are as follows:

- Improve the current community care service system

- by using agent-oriented engineering solutions to design new style cooperative, coordinated, and collaborative health care services via internet.

- by sharing services, distributed resources and decision making;

- by requiring the communication of complex and diverse forms of flowing information between each individuals such as a variety of clinical and other settings;

- by the coordination abilities between groups of health care professionals with diversity roles, skill and responsibility;

- by the interactive and cooperative effectiveness in the health care environment, in order to meet the information needs of the patients and health care providers;

- Quickly contact the help agent-based services that you need during the community care events. Promoting effective and coordinative delivery of health care services by using a range of agents to communicate with available healthcare professions. The patients information to be added and discussed through the use of mediators $[9,11]$ and agent-based negotiation strategies and decision-making system.

- Improve coordination between social services and emergency services, organizing and managing these concurrent actions in an effective and inteoperative way.

- Link effectively and increase the speed of response and supports (such as emergency assistance services)by connecting and communicating with autonomy intelligent agents and an agent-based health care environment

- The provision of positive assistance to maintain and enhance the quality of service and the provision of routine service as specified by the Individual Service Plan and provided to an individual on a routine basis to against crisis. The elimination of unnecessary duplication of resources.

\section{THE Strengths of Agent-BASEd MODELS FOR SYSTEMS' ANALYSIS AND DESIGN}

The main task of agent-based models is the attempt at making the design and development of community healthcare system as easily as possible. By using agentbased models could reduce design effort and increase productivity, since it provides a powerful high-level abstraction for agents' analysis and design and resource management; it defines the process of development with clear reliable, flexible phases. Therefore, it greatly simplifies the configuration and organization of agents and services, the endowment of communication capability as well as cooperation capability of agents, and provides a better understanding of the system and its connected working environment.

In summary of the strengths of the agent-based models for community care systems analysis and design as we discovered have numerous positive characteristics including:

- It marks the derivation of macro-level descriptions from the problem specification, then mapping of the macro-level description into the micro-level interaction specifications by using adequate operation and formulisation; It provides a set of guidelines whereby designers and developers are involved in the whole planning process from information collection to development of action plans and management.

- It handles the complexity of the software development process increasing the quality of the resulting system. Nowadays, as agents become more sophisticated they need to interact in more sophisticated ways and interactions may require several communications to complete tasks. Each task within a design describes a set of specific aspects of the system under consideration [13,14 ]. It is very clear that we have to use a set of models for controlling overall conditions and agent behaviours. The agents' domain dynamic behaviors should also be considered as being the same as the static aspect in an agent environment. Obviously, within a largescale distributed agent-based system, the difficulties of managing their development process will be dramatically increased as well as dealing with the variable behaviors of the agents. The design of those kinds of complex systems involves an incremental and interactive process.

- It attempts to be a practical approach to the analysis, design, implementation, and management of agent 
software systems, since it provides a range of models for addressing agents, services, resources, and their community and interaction.

- It delineates between generic agent-level and domain-level problem-solving issues. It helps developers see the involvement of agents and understand the work of multi-level development phases and how decompose complex domain problems into small pieces. Each design stage contains different mechanism for identifying roles and function setting, and then producing a control and action rules to run a control-level process associated with a domain action. It is the most important consideration that a designer should include enough information. By using agent-based design models and tools can clearly clarify roles and tasks for each agent, and each agent could be easily identified and described, and later on be organised into an appropriate agent architecture.

- It provides an engineering approach to the design and construction of collaborative agent-based computer systems. It helps system developers to focus on providing a formal platform for analyzing, designing, organizing, controlling and managing agents' behaviour. It offers high-abstraction level specifications and a range of capable structural models for systems analysis and design. It offers practical support and guidance, which currently only covers system analysis and design phases, while proving a range of guidelines that are directly relevant to agents in their coordinative working domain with built-in agent convention roles or models for agent inter-connections and interactions in common highly flexible agent-based computer environments.

Those efforts have resulted in a number of architectures and frameworks for agents, but there still has been relatively little systematic research and few real-life applications, which could fully address the issues of practical methods for the design of agent-based systems in dynamic and distributed environments.

\section{CONCLUSIONS}

This paper shows that using our newly developed agentbased modelling techniques, models and tools can assist the development of agent-based systems and environments to further incorporate system creation within an agent domain.

The exploration of a real system (e.g. agent-based community health care system) and its modelling techniques for agent-based computer systems is not only allowing us to find more effective ways of understanding and organizing agent environments, but also giving us a good opportunity to in-depth discovering some useful models for design and development of collaborative agentoriented architectures and agent-based applications. As a result of this, we could face the challenges and pressures of ever greater complexity of the software systems' creation and development. An advanced and effective community care systems can be developed and implemented very soon.

\section{REFERENCES}

[1] Beer, M.D, Huang, W., \& Sixsmith A.(2002), in name of book Intelligent Agent and Applications, Chapter 10, Springer-Verlag, Heidelberg, pp.307-354.

[2] Beer, M.D., Hill, R., Huang, W. and Sixsmith, A.(2003), Using Agents to Promote Effective Co-ordination in a Community Care Environment. Chapter 3, in Ye, Y.and Churchill, E (Eds): Agent Supported Collaborative Work, Kluwer Academic Publishers, USA, pp.53--78.

[3] Huang. W. \& Beer, M.D. (2001) "Towards Intelligent Health Care System, an introduction to Agent-based Community Care System --INCA", paper in conference proceedings of PERP 2001, The University of Keele, UK, pp.95--99.

[4] Huang.W(2002), Developing Advanced Agent Systems with UML and Gaia Methodology. Proceedings of the $6^{\text {th }}$ World Multiconference on Systemic, Cybernetics, and Informatics (SCI2002), pp 97-106.

[5] Huang,W.\&Beer,M.D.(2003), Community Care System Design and Development with AUML. Proceedings of the 9th International Conference on Information Systems Analysis and Synthesis ISAS '03), pp 247-253

[6] Jennings, N.(1999), An Agent-based Approach for Building Complex Software Systems. Communications of The ACM, Vol.44 No4, pp.35-41.

[7] Jennings, N.R(2003), Agent-based Control System: why are they suited to engineering complex systems? IEEE. Control Systems Magazine 0272-1708/03\%. June 2003 pp.61-73

[8] Object Management Group (1999), "OMG Unified Modelling Language specification version 1.3", June 1999.

[9] Peckham, M and Smith, R.(1996), Health Care for an Aging Society. Churchill Living Stone.

[10] Priestley. M (2000), "Practical Object-Oriented Design with UML" MeGraw-Hill Companies, Inc, 2000. pp 5-7.

[11] NHS (1998), Information for health: An Information Strategy for the Modern. NHS Guidance on Implementing Information for Health, Department of Health Document HSC 1998/168, London, UK September 1998

[12] Sommerville,I.(2004), Software Engineering, seventh edition, Addison-Wesley Publisher Ltd, England.

[13] Tecuci,G.(1998), Building Intelligent Agents: An Apprenticeship Multistrategy Learning Theory, Methodology, Tool and Case Studies, Academic Press, California, USA.

[14] Van Dyke Parunak, H. and Odell, J. (2002), "Representing Social Structures in UML," Agent-Oriented Software Engineering Workshop II, Michael Wooldridge, Paolo Ciancarini, and Gerhard Weiss, eds., Springer, Berlin, 2002, Canada, May 2001, pp. 1-16.

[15] Wooldridge, M.J.\&Jennings, N.R.ed.(1995), Intelligent Agent: Theory and Practice. Journal of The Knowledge Engineering Review 10(2), pp. 115-152

[16] Wooldridge, M.J, Jennings, N.R and Kinny, D.(2000), The Gaia Methodology for Agent-Oriented Analysis and Design. Journal of Autonomous Agents and Multi-Agent Systems. 3(3):285-312. 